\title{
Resistance to Rocuronium in a Patient with Propionic Acidemia Undergoing Appendectomy for Acute Appendicitis: A Case Report with Literature Review
}

\author{
Jurij Janežl, ${ }^{\star \star}$, Armand Dominik Škapin ${ }^{2}$
}

\author{
${ }^{1}$ University Medical Centre Ljubljana, \\ Slovenia and University of Ljubljana, \\ Faculty of Medicine, Slovenia \\ ${ }^{2}$ University Medical Centre Ljubljana, \\ Slovenia

\section{*Correspondence} \\ jurij.janez@kclj.si \\ (Jurij Janež)
}

\begin{abstract}
Propionic acidemia is a rare metabolic disorder caused by a deficiency of the mitochondrial enzyme, propionyl-CoA carboxylase, which is required in the catabolic pathways of several amino acids, odd-chain fatty acids and the side chains of cholesterol. Clinically, the disorder may present with multisystemic manifestations including metabolic ketoacidosis, hyperlactatemia, hyperammonemia, developmental delay and neurological dysfunction. The key principle in managing or preventing acute exacerbations consists of protein restricted diet and avoidance of factors that may potentiate relevant catabolic pathways. We describe an unusual case of a female patient with propionic acidemia undergoing appendectomy for acute appendicitis, who was resistant to a nondepolarizing neuromuscular blocker (rocuronium). In literature, there has been reported cases of resistance to nondepolarizing neuromuscular blockers associated with certain pathologic conditions or medications, but such resistance has not been previously described in the context of propionic acidemia. Rocuronium resistance in our index patient could be attributable to a sum of potential etiologic factors including the use of valproic acid, and upper motor neuron damage, a rare complication of chronic propionic acidemia.
\end{abstract}

\section{Keywords}

Propionic academia, Nondepolarizing muscle relaxants, Rocuronium, Appendicitis, Anesthesia

\section{INTRODUCTION}

Propionic acidemia is an inherited autosomal recessive, metabolic disorder, caused by a deficiency of the mitochondrial enzyme, propionyl-CoA carboxylase (PCC), which results in the accumulation of toxic metabolites, such as propionic acid [1]. The most common clinical manifestations include metabolic acidosis, hyperammonemia, poor feeding, lethargy, vomiting, osteoporosis, neurological dysfunction, bone marrow suppression, developmental retardation and cardiomyopathy [2]. The overall estimated incidence is around 1 in 100.000 to 150.000 [3]; however, in some populations across the world, the incidence is much higher (e.g. in Saudi Arabia) [3]. Patients with propionic acidemia undergoing any major surgical procedures should be treated according to a special perioperative plan [4]. Therefore, these patients present a unique challenge for a surgical team, especially for the anesthesiologist. It is recommended that all elective operations in such patients should take place in a tertiary center with access to a multidisciplinary team and an intensive care unit. However, there is a lack of published material on the management of patients with propionic acidemia in need of an emergency surgery. This paper presents a case of an 18-year old female patient with propionic acidemia referred to our hospital for an emergency operation for acute appendicitis, who was resistant to a nondepolarizing neuromuscular blocker (rocuronium).

\section{CASE REPORT}

An 18-year old female patient with known propionic acidemia and epilepsy was referred to our surgical department on account of acute appendicitis, confirmed 
radiologically with abdominal ultrasound (US). She also suffered from developmental delay and hypothyroidism, and her past surgical history was remarkable for a gastrostomy created under local anesthesia to facilitate feeding and medication administration. Her long-term drug therapy consisted of L-carnitine, sodium benzoate, metronidazole (for 2 weeks with 3 weeks off), sodium valproate, levothyroxine and pantoprazole along with a low-protein diet $(0.9 \mathrm{~g} / \mathrm{kg} /$ day $)$ with a non-propiogenic amino acid mixture and Coenzyme Q10. Her weight was $62 \mathrm{~kg}$ with $41 \mathrm{~kg}$ of lean body mass, her height was 151 $\mathrm{cm}$ and her BMI was 27.2.

The patient presented with episodic pain in the right lower abdomen, and vomiting. She was initially managed conservatively in a pediatrics department for one day without satisfactory clinical improvement, necessitating a referral to our surgical unit. Upon admission in our department, an urgent abdominal computed tomography (CT) was performed, which did not show any pathological findings (diameter of appendix was 7 millimeters). However, on the next day, the symptoms deteriorated, with a significant elevation in C-reactive protein (CRP) and leucocytes. An urgent abdominal ultrasound was then performed, which revealed an inflamed appendix. The preoperative blood test values were: blood glucose $8.2 \mathrm{mmol} / \mathrm{L}$; creatinine 58 $\mu \mathrm{mol} / \mathrm{L}$; ammonium ion $28 \mu \mathrm{mol} / \mathrm{L}$; $\mathrm{pH} 7.46$; bicarbonate $21.4 \mathrm{mmol} / \mathrm{L}$; lactate $2.97 \mathrm{mmol} / \mathrm{L}$.

During fasting period, the patient was maintained on intravenous infusion of $15 \%$ glucose at $120 \mathrm{~mL} / \mathrm{h}$ together with $0.01 \mathrm{E} / \mathrm{kg} / \mathrm{h}$ of insulin. Before the patient was moved into an operating theatre, she received a bolus L-carnitine. In the theatre, she was placed in a supine position and received intravenous perioperative antibiotic prophylaxis. During the operation the intravenous infusion of $15 \%$ glucose was raised to $170 \mathrm{~mL} / \mathrm{h}$ and insulin infusion was being adjusted according to blood glucose levels measured every 30 minutes. Induction of anesthesia was performed with sufentanil ( $5 \mu \mathrm{g}$ at induction and $40 \mu \mathrm{g}$ all together), propofol (100 $\mathrm{mg}$ at induction) and rocuronium (total dose of $70 \mathrm{mg} ; 30 \mathrm{mg}$ at induction and $40 \mathrm{mg}$ in smaller doses during the operation). The patient's Mallampati score was III. After the induction dose of rocuronium there was no obvious loss of tone in the throat muscles. Accordingly, intubation was difficult to perform. It was eventually achieved on the fourth attempt. For induction and maintenance of anesthesia the anesthesiologist used sevoflurane $(77.32 \mathrm{ml}$ used throughout the operation). Other anesthetic medications used included: midazolam (2 $\mathrm{mg}$ before induction), sugammadex (100 mg), metamizole (2.5 g after operation) and droperidol (2.5 $\mathrm{g}$ after operation).

We started the operation with a periumbilical incision and inserted a laparoscopic trocar using the Hasson open technique. The attempt to create pneumoperitoneum failed; the intra-abdominal pressure jumped to $14-15 \mathrm{mmHg}$ as soon as we connected the gas line to the trocar. Nevertheless, we inserted a camera through the trocar, but visibility was poor. The abdominal wall was hard and tense even though the patient had received an initial dose of the muscle relaxant (rocuronium). She was therefore administered additional dose of the muscle relaxant, but the patient's abdominal wall still remained rigid. Because the conditions made it impossible to continue the operation laparoscopically, we decided to perform a conversion surgery. Throughout the laparoscopic part of the operation intraabdominal pressure was at $14-15 \mathrm{mmHg}$ at all times. We made a lower median laparotomy and entered the abdominal cavity. However, the patient was still not relaxed, intestines were protruding out through the laparotomy and there was poor visibility in the abdomen. With difficulty, we found a retrocecal gangrenous appendix and performed a classic appendectomy. It was not possible to bury the appendiceal stump into the base of the cecum, because the visibility of the appendiceal base was poor. Abdominal drain size 18 was placed into the pelvis from the right side of the abdomen. Hemostasis was complete at the end of the operation. We irrigated the abdominal cavity with saline and closed the laparotomy with simple interrupted sutures. Patient was extubated without any problems. The patient's body temperature was measured before and after the operation, it was $36.8^{\circ} \mathrm{C}$ and $36.6^{\circ} \mathrm{C}$, respectively. During the operation her heart rate was between 120-140 beats/min, systolic blood pressure was between $80-110 \mathrm{mmHg}$, diastolic blood pressure was between 45-65 $\mathrm{mmHg}$ and her oxygen saturation levels were between $92-95 \%$. There were no complications in the postoperative period. She remained in the post-anesthesia care unit for 65 minutes.

For the next two postoperative days the patient remained under observation in abdominal surgical ward, in intensive care unit. She was treated according to the guidelines for management of propionic acidemia. The patient's postoperative stay in our department was uneventful, and she was subsequently transferred back to the pediatrics department. Abdominal ultrasound performed three days after the operation showed a six by three centimeters large abscess collection in the right lower abdomen (a possible consequence of the failure to bury the appendiceal stump into the cecum), which gradually regressed over the following two weeks with antibiotics.

\section{DISCUSSION}

Propionyl-CoA carboxylase (PCC) catalyzes the biotindependent conversion of propionyl-CoA to methylmalonylCoA, which enters the Krebs cycle via succinyl-CoA. Sources of propionate are branched chain amino acids (leucine, isoleucine, valine, threonine and methionine), odd-chain fatty acids and the side chains of cholesterol $[3,5]$. PCC is essential for the catabolism of these metabolites. In cases of deficient activity of PCC, there is an accumulation of propionyl-CoA and consequently 3-OH-propionic acid, methylcitrate, propionylglycine and other metabolites $[3,5]$. Accumulation of these potentially toxic metabolites can cause severe clinical manifestations in patients and presents the main problem of propionic acidemia. 
The disorder is characterized by recurrent exacerbations which occur when there is increased catabolic activity (e.g. infection, excessive protein intake, constipation, physical exercise, certain medications). The increased catabolic activity leads to a rapid production and accumulation of toxic metabolites because of the enzyme deficiency. Exacerbations present clinically with metabolic ketoacidosis, hyperlactatemia and hyperammonemia $[2-4,6]$. Ketoacidosis is caused by propionic acid inhibiting citric acid cycle enzymes (Krebs cycle) [2, 4] and hyperammonemia develops because propionic acid inhibits synthesis of $\mathrm{N}$ acetylglutamate, one of the enzymes in the urea cycle $[4,6]$.

Patients with a complete enzyme deficiency present in the first days to weeks of life with acute deterioration, hyperammonemia and metabolic acidosis, progressing to coma and death without appropriate treatment. On the other hand, late-onset disease can present at any age with a more diverse clinical picture [1, 3, 6]. Clinical signs and symptoms of propionic acidemia are nonspecific and can affect different organ systems. Acute presentation includes sepsis-like picture, temperature instability, respiratory distress, vomiting, altered level of consciousness, movement disorders and pancytopenia. Chronic progression of the disease can lead to developmental delay, movement disorders, hypotonia, episodes of vomiting with ketoacidosis, failure to thrive, anorexia, pancytopenia and cardiomyopathy [3].

The most commonly used tests to detect propionic acidemia are determination of organic acids in urine and analysis of acylcarnitine profile in blood. Additional confirmation can be done by enzyme assay in cultured skin fibroblasts and molecular genetic analyses [1, 3, 4].

Treatment of propionic acidemia can be divided into acute management and chronic, long-term management. The goal of acute management is to reverse acute metabolic crisis and to reduce hyperammonemia. We achieve that by reversal of catabolism and by removal of toxic metabolites. At first, decompensated patients should be stabilized, all protein intake should be restricted and intravenous glucose should be started. The patients should also receive Lcarnitine (it conjugates with propionate and promotes its movement out of cells, so it can be excreted in urine), sodium benzoate (nitrogen scavenger), sodium bicarbonate, non-absorbable oral antibiotic (metronidazole; a lot of propionate is produced by bacteria in the intestine) and biotin (PCC is a biotin-dependent enzyme). Other treatments that can sometimes be useful include insulin, Larginine (promotes ammonia excretion through the urea cycle), hemodialysis and antimicrobial therapy. Proteins should be reintroduced in 24-36 hours after the restriction $[1,3]$. On the other hand, the goals of the long-term management are to achieve normal development of patients and to prevent metabolic decompensations. One of the most essential things in long-term management is a low-protein diet with non-propiogenic amino acid mixture, which can also be administered through nasogastric or gastrostomy tube if necessary. The amount of protein $(\mathrm{g} / \mathrm{kg} / \mathrm{day})$ in a low-protein diet is determined by age, growth, severity of condition and metabolic stability [3]. Other recommended treatment includes regular use of carnitine, sodium bicarbonate and non-absorbable oral antibiotic [1,3]. Most of the metabolism involving PCC occurs in the liver, therefore, liver transplant [2, 3, 7].

There are various considerations regarding anesthetic management of patients with propionic acidemia. The anesthesiologist's main concern is to avoid events that could precipitate metabolic acidosis in these patients (catabolism, hypoxia, dehydration, hypotension and the use of inappropriate anesthetics) [2, 4, 6]. To suppress catabolism during preoperative fasting period, patients require glucose in their intravenous fluids. Some patients with propionic acidemia can be prone to vomiting due to abnormal gag reflex; therefore, a rapid sequence induction of anesthesia is recommended. Lactated Ringer's solution should be avoided because lactate can contribute to acidosis $[2,6]$. Anesthesiologists should furthermore avoid drugs that are metabolized directly to propionic acid, odd-chain organic acids, odd-chain alcohols, acrylic acid and odd-chain fatty acids. These molecules are further metabolized to propionic acid and can thus precipitate metabolic acidosis. Such drugs that should be avoided include: muscle relaxants like succinylcholine, atracurium, cisatracurium and mivacurium; propofol containing soybean oil rich in polyunsaturated fats; and analgesics including ibuprofen, naproxen and ketoprofen [2, 4]. Basic principles of perioperative anesthetic management of patients with propionic acidemia don't differ between elective and emergency surgical procedures.

In our case, the long-term management of the patient was fairly successful with a low number of exacerbations. Her major long-term complications were developmental delay and epilepsy, which are common in this disorder [3]. The patient's treatment was in accordance with the standard recommendations and included a low-protein diet, which was mostly administered through a gastrostomy tube. Additionally, she was receiving sodium valproate for her epilepsy and levothyroxine for her hypothyroidism. Sodium valproate is contraindicated in propionic acidemia because it decreases L-carnitine concentration in plasma and needs to be used with great caution and when there are no other antiepileptic drug alternatives [3].

The diagnosis of acute appendicitis was difficult to make at the beginning since her symptoms were nonspecific and could also be a result of an exacerbation of propionic acidemia. Besides, the initial diagnostic imaging showed unremarkable findings. Since she was unable to feed orally, she was maintained on intravenous glucose with insulin even before the operation, as part of the preventive measures to avoid triggering any exacerbations. Although propofol was used in the induction of anesthesia, it is not recommended for use in patients with propionic acidemia because its aqueous solution also contains soybean oil, which is high in polyunsaturated fats and a small amount of them can be metabolized to propionic acid [2, 4]. However, we didn't notice any serious consequences because of its use, possibly on the account of the patient having received 
L-carnitine even before the operation, and only a small amount of polyunsaturated fats potentially metabolizing to propionic acid. Other drugs that were used during anesthesia are not contraindicated and are safe to use. Intubation was difficult to perform because of unrelaxed throat muscles and abnormal gag reflex. Hyperactive gag reflex is common in patients with propionic acidemia $[3,6]$, but could also be a consequence of ineffective muscle relaxant.

Perhaps, the most interesting aspect of this case was the patient's resistance to rocuronium, an aminosteroid nondepolarizing neuromuscular blocker, which is considered safe for use in patients with propionic acidemia [2, 6]. The resistance wasn't recorded with neuromuscular transmission monitoring (e.g. train-of-four monitoring) because in our institution neuromuscular transmission monitoring is not routinely used in emergency operations. Such monitoring would have been useful as it can optimize the dosage and timing of both neuromuscular blocker and reversal agent administration [8]. The anesthesiologist started with a 30 $\mathrm{mg}$ bolus of rocuronium at induction of anesthesia and used another $40 \mathrm{mg}$ in smaller doses during the surgery. The recommended initial dose of rocuronium for normal induction for our patient weighing $62 \mathrm{~kg}$ is $37 \mathrm{mg}(0.6$ $\mathrm{mg} / \mathrm{kg}$ ). Although the patient received a little less than the recommended stat dose, it is known that even a somewhat lower dose should have a comparable effect [9]. The anesthesiologist didn't perform rapid sequence intubation which requires a higher dose of rocuronium (1-1.2 mg/kg) because he wasn't aware it is recommended in patients with propionic acidemia. Maintenance dosing of rocuronium is primarily based on the clinical duration following previous dose (initial or prior maintenance dose) and should not be administered until the recovery of neuromuscular function is evident. Maintenance doses vary from $0.1-0.2 \mathrm{mg} / \mathrm{kg}$, calculated to 6-12 mg for our patient. Therefore, maintenance doses of $40 \mathrm{mg}$ summed together should be enough for a 70-minute operation. We can conclude that the used dosage of muscle relaxant was appropriate.

The main mechanism of resistance to neuromuscular blockers is upregulation of acetylcholine receptors in skeletal muscles. (The nicotinic acetylcholine receptors on motor endplates are the target of neuromuscular blockers). Some pathological states that exhibit such resistance are upper and lower motor neuron lesions, thermal injury, disuse atrophy, direct muscle trauma, chronic exposure to nondepolarizing agents and chronic use of antiepileptic drugs [10-12]. An additional mechanism causing resistance to neuromuscular blockers in chronic use of anticonvulsants is induction of cytochrome P450 enzyme system. This system is in part responsible for the neuromuscular blockers' elimination [10, 12, 13]. Additionally, some pharmacokinetic factors were found to impact the effects of nondepolarizing neuromuscular blockers (e.g. changes in volume of distribution, changes in clearance, changes in plasma protein binding) [14].

Resistance to the muscle relaxant in our patient could be due to propionic acidemia, mindful that upper motor neuron damage with associated spasticity is one of the rarer complications of propionic acidemia [3]. (Neurological complications in general are, however, quite common in the disorder). Damage to the upper motor neurons leads to lower inhibition with resultant hyperactivity of lower motor neurons. In such patients, there is an increased resistance to the effects of neuromuscular blockers (including rocuronium) [10, 11]. Accordingly, a standard dose of a muscle relaxant may not necessarily be enough to negate the increased activity of the lower motor neuron. In our patient, damage to the upper motor neuron is very likely, although perhaps not substantial enough to be evidenced clinically. Such potential subclinical damage may however, be sufficient to counteract the effects of the muscle relaxant used. Another factor that can decrease the effects of the muscle relaxant is the use of valproic acid as valproic acid has a minor drug interaction with rocuronium [15]. In the light of these considerations, our overall inference is that that the ineffectiveness of the muscle relaxant is likely a consequence of multiple factors resisting or lowering its pharmacodynamic effects. Meanwhile, given that this was our patient's first surgery under general anesthesia, we do not have any prior knowledge regarding the effectiveness of muscle relaxants in different clinical circumstances.

\section{CONCLUSION}

Propionic acidemia can cause various complications and requires unique acute and chronic management principles. Because of the rarity of the disease, many physicians are not familiar with it, hence diagnosis and proper management remains a conundrum in clinical practice. It is compelling, however, that clinicians, especially anesthesiologists, are more aware of the condition and accordingly exercise necessary caution in order to avoid any exacerbations during general anesthesia. The index case was unique for our team, and to the best of our knowledge, it is the first reported case of resistance to a nondepolarizing neuromuscular blocker in a patient with propionic acidemia. Most likely this resistance was a consequence of multiple factors summed together, and a long-term complication of propionic acidemia could be one of them.

\section{ACKNOWLEDGEMENTS}

Thank numerous individuals participated in this study.

\section{CONFLICT OF INTEREST}

The authors declare that they have no conflict of interest.

\section{ETHICS APPROVAL}

Ethics approval is not needed for case reports in our institution as per their instructions. 


\section{STATEMENT OF INFORMED CONSENT}

Informed consent was obtained from the patient's parents for publication of this case report.

\section{REFERENCES}

[1] Rafique M. Emerging trends in management of propionic acidemia. Arq Bras Endocrinol Metabol. 2014;58:237-42.

[2] Ryu J, Shin YH, Ko JS, Gwak MS, Kim GS. Intractable metabolic acidosis in a child with propionic acidemia undergoing liver transplantation - a case report. Korean J Anesthesiol. 2013;65:25761 .

[3] Baumgartner MR, Hörster F, Dionisi-Vici C, Haliloglu G, Karall $\mathrm{D}$, Chapman KA, et al. Proposed guidelines for the diagnosis and management of methylmalonic and propionic acidemia. Orphanet $\mathrm{J}$ Rare Dis. 2014;9:130.

[4] Harker HE, Emhardt JD, Hainline BE. Propionic acidemia in a fourmonth-old male: a case study and anesthetic implications. Anesth Analg. 2000;91:309-11.

[5] Soberón JR, Elliott CE, Bland KS, Weinberg GL. Peripheral nerve block in a patient with propionic acidemia. Reg Anesth Pain Med. 2014;39:560-1.

[6] Sebens EL. A child with propionic acidemia undergoing dental restorations: a case report. AANA J. 2011;79:295-299.

[7] Silva HM, Nassogne MC, Smets F, Stéphenne X, Scheers I, Veyckemans F, et al. Liver Transplantation for Propionic Acidemia. J Pediatr Gastroenterol Nutr. 2017;64:e73-e76.

[8] Deana $\mathrm{C}$, Barbariol F, D'Incà S, Pompei L, Rocca GD. SUGAMMADEX Versus Neostigmine After ROCURONIUM Continuous Infusion in Patients Undergoing Liver Transplantation. BMC Anesthesiol. 2020;20:70

[9] Fuchs-Buder T, Schlaich N, Ziegenfuss T. [Rocuronium for anes- thesia induction in elective procedures. Time course of muscular blockade and intubation after administration of 2-compartment ED95 $(0.6 \mathrm{mg} / \mathrm{kg})$ and dose reduction $(0.4 \mathrm{mg} / \mathrm{kg})]$. Anaesthesist. 1999;48:164-8.

[10] Martyn JA, White DA, Gronert GA, Jaffe RS, Ward JM. Upand-down Regulation of Skeletal Muscle Acetylcholine Receptors. Effects on Neuromuscular Blockers. Anesthesiology. 1992;76:82243.

[11] Capuano A, Sullo MG, Rafaniello C, Sportiello L, Fusco P, De Vizia $\mathrm{M}$, et al. Complete resistance after maximal dose of rocuronium. J Pharmacol Pharmacother. 2015;6:175-178.

[12] Soriano SG, Martyn JA. Antiepileptic-induced Resistance to Neuromuscular Blockers: Mechanisms and Clinical Significance. Clin Pharmacokinet. 2004;43:71-81.

[13] Anderson GD. A Mechanistic Approach to Antiepileptic Drug Interactions. Ann Pharmacother. 1998;32:554-63.

[14] Lien CA, Eikermann M. 22 - Neuromuscular Blockers and Reversal Drugs. In: Hemmings HC, Egan TD, editors. Pharmacology and Physiology for Anesthesia, 2nd ed. Philadelphia, PA: Elsevier, Inc. 2019.p.428-454.

[15] Kim MH, Hwang JW, Jeon YT, Do SH. Effects of valproic acid and magnesium sulphate on rocuronium requirement in patients undergoing craniotomy for cerebrovascular surgery. $\mathrm{Br} \mathrm{J}$ Anaesth. 2012;109:407-12.

How to cite this article: Jurij Janež, Armand Dominik Škapin. Resistance to Rocuronium in a Patient with Propionic Acidemia Undergoing Appendectomy for Acute Appendicitis: A Case Report with Literature Review. Signa Vitae. 2020;16(1):187-191. doi:10.22514/sv.2020.16.0026. 